\title{
CoRoT data contribution to stellar seismology
}

\author{
E. Michel, ${ }^{1}$ A. Baglin, ${ }^{1}$ R. Samadi, ${ }^{1}$ F. Baudin, ${ }^{2}$ M. Auvergne ${ }^{1}$ \\ ${ }^{1}$ Observatoire de Paris, LESIA, UMR 8109, pl. J. Janssen, 92195 Meudon, France \\ ${ }^{2}$ Institut d'Astrophysique Spatiale, CNRS/Univ. Paris XI, UMR 8617, 91405 Orsay, France
}

\begin{abstract}
At the time to submit papers for these proceedings, CoRoT will be launched in less than one month. The scientific programme, the instrument and the mission profile have been described in several places recently (e.g., Baglin et al. 2006, Michel et al. 2006a) and a dedicated volume (Fridlund et al. 2006) has been published, where these aspects are commented on in detail. In the present paper, we focus on a description of the nature and quality of the data expected from the CoRoT seismology observational programme.

We thus first review a few specific aspects of the CoRoT instrument and mission profile necessary to have a clear idea of the nature and quantity of the data to come. Then, we produce data simulations for selected targets to illustrate the expected performance. In particular, we consider classical pulsators, extending the work initiated by Michel et al. (2006a) with solar-like pulsators.
\end{abstract}

\section{The instrument - main outline}

The instrument collects light through an off-axis telescope plus a dioptric objective giving access to a $3 \times 2.7$ degrees field of view for a $588 \mathrm{~cm}^{2}$ collecting area, equivalent to a $27 \mathrm{~cm}$ aperture. The focal plane hosts four CCDs ( $2 k$ by $4 k$ pixels) used in frame-transfer mode.

Half of the field (two CCDs) is mainly devoted to the seismology programme of CoRoT. It is defocused (diameter of the star spot $\sim 18 p x$, i.e. $\sim 41$ arcsec) and 10 target stars with $5.4<m_{V}<9$ can be observed simultaneously with a 1 second sampling time. By default, ten windows are read for targets and ten for background estimates. On-board realtime photometry is achieved. Part of these target window images can be downloaded for further refined analysis ( 6 to 10 among 10 with a 32 seconds sampling).

The "exoplanet field" is in focus and it is possible to observe 12000 targets with $11<$ $m_{V}<16$ at a sampling time of $512 \mathrm{~s}$. Thanks to a prism put on the exo-field, each target image is slightly dispersed and for the brightest objects $\left(m_{V}<14.5\right)$ three-colour information (white-blue-red) can be obtained. For a limited number of targets $(\sim 500)$, a higher sampling rate can be used $(32 \mathrm{~s})$.

\section{Mission profile}

CoRoT's mission profile is characterized by the possibility to dedicate long runs (up to $150 \mathrm{~d}$ ) to a specific field. The price for this is that CoRoT observations are restricted to two observing zones defined as cones of 10 degrees on the sky, around Position C, roughly in the Galactic centre direction $\left(\alpha=18^{\circ} 50, \delta=0^{\circ}\right)$ and its opposite: Position A, roughly in the Galactic anticentre direction $\left(\alpha=6^{\mathrm{h}} 50, \delta=0^{\circ}\right)$.

The mission profile is thus built around successive $150 \mathrm{~d}$ long runs, alternatively in the centre and anticentre directions, separated by short runs ( $\sim 3$ weeks) also in one of these accessible cones. 
The observing program will start with an Initial Run (IR1) in the anticentre direction. Its duration is expected to be between 60 and 80 days, depending on the final operational schedule. The list of targets selected for this run features in particular: a solar-like pulsator on the Main Sequence, a known $\delta$ Scuti pulsator, two Am stars, two eclipsing binaries (one including a B5 star and the other an A0 star), an Ap star, and two giant stars.

After this Initial Run, by mid-April, the satellite will be flipped by 180 degrees to observe the first Long Run field in the centre direction (LRC1). The selection of this field has been driven mainly by two known classical pulsators: a $\beta$ Cephei and a $\delta$ Scuti star, but it also features two solar-like pulsator candidates: a relatively bright one $\left(m_{V}=6.6\right)$ illustrative of the good candidates (criterion 2 as defined by Michel et al. 2006b) and the other one, fainter $\left(m_{V}=7.7\right)$ being illustrative of criterion 0 (see Michel et al. 2006b), i.e. candidates for which detection of a significant number of modes is expected but with no guarantee on the precision of measured frequencies.

After this first Long Run, a Short Run (SRC1) is planed in the same direction with a duration of 20 days. Then, around mid-October, the satellite will be flipped again to point to the Long Run LRA1, in the anticentre direction during 150 days, followed by a short run SRA1, and so on and so forth. The mission is planned for 3 years, but in the nominal scenario, there is no technical limitation to an extension.

A preliminary observational programme has been settled for the first years of the mission (see Michel et al. 2006c). This list of objects which will be observed during 150 days in the four first Long Runs features: one O9 star, eleven B stars, including one known $\beta$ Cephei and five Be stars, eleven $\mathrm{A}$ stars including two known $\delta$ Scuti pulsators (one being in an eclipsing binary) and two Ap stars, fourteen $\mathrm{F}$ stars including six solar-like candidates (one known solar-like pulsator), one $\delta$ Scuti star, one $\gamma$ Doradus star, and three G stars including two solar-like candidates (one with a known planet).

The two next Long Runs are known, but the definitive position and the list of targets is not settled yet. A projection of what the list could be after the 6 first Long Runs and the Initial Run is given in Fig. 1. As shown in Fig. 1, this sample gathers a significant set of objects scanning the Main Sequence and post Main Sequence stage for a large range of mass.

\section{Photometric performance in the Seismo field}

In this field, and in the range $[0.1 \mathrm{mHz}, 10 \mathrm{mHz}]$, the instrumental noise has been kept below the photon noise, in the range of magnitude $5.4<\mathrm{m}_{V}<9-9.5$, except for a few harmonics of the orbital period (at $\omega_{0}=162 \mu \mathrm{Hz}, 2 \omega_{0}, 4 \omega_{0}, 5 \omega_{0}$ ) which will be kept as low as possible by corrections.

In this context, the photometric precision obtained with CoRoT is $\sigma=0.6 \mathrm{ppm}$ in 5 days for a $m_{V}=5.7$ target $\left(\sigma_{1 s} \sim 4 \times 10^{-4}\right.$ and $\left.0.156 \mathrm{ppm}^{2} / \mu \mathrm{Hz}\right)$. For a $m_{V}=9$ target, these numbers become $\sigma=2.75 \mathrm{ppm}$ in 5 days $\left(\sigma_{1 \mathrm{~s}} \sim 1.8 \times 10^{-3}\right.$ and $\left.3.26 \mathrm{ppm}^{2} / \mu \mathrm{Hz}\right)$.

\section{Performance in terms of eigenfrequency measurements}

In order to illustrate the expected performance in terms of precision on frequency measurement, we complete and develop the work presented by Michel el al. (2006a). We use the simulation tool Simu-LC developed in the framework of the CoRoT Seismology Working Group (http://www.lesia.obspm.fr/ corotswg) and described by Baudin et al. (2006). This tool takes into account granulation noise estimates following Harvey (1985) and photon noise in the CoRoT framework. For solar-like pulsators, it also takes into account theoretical mode excitation rates following Samadi \& Goupil (2001) and theoretical mode damping rates from Houdek et al. (1999). 


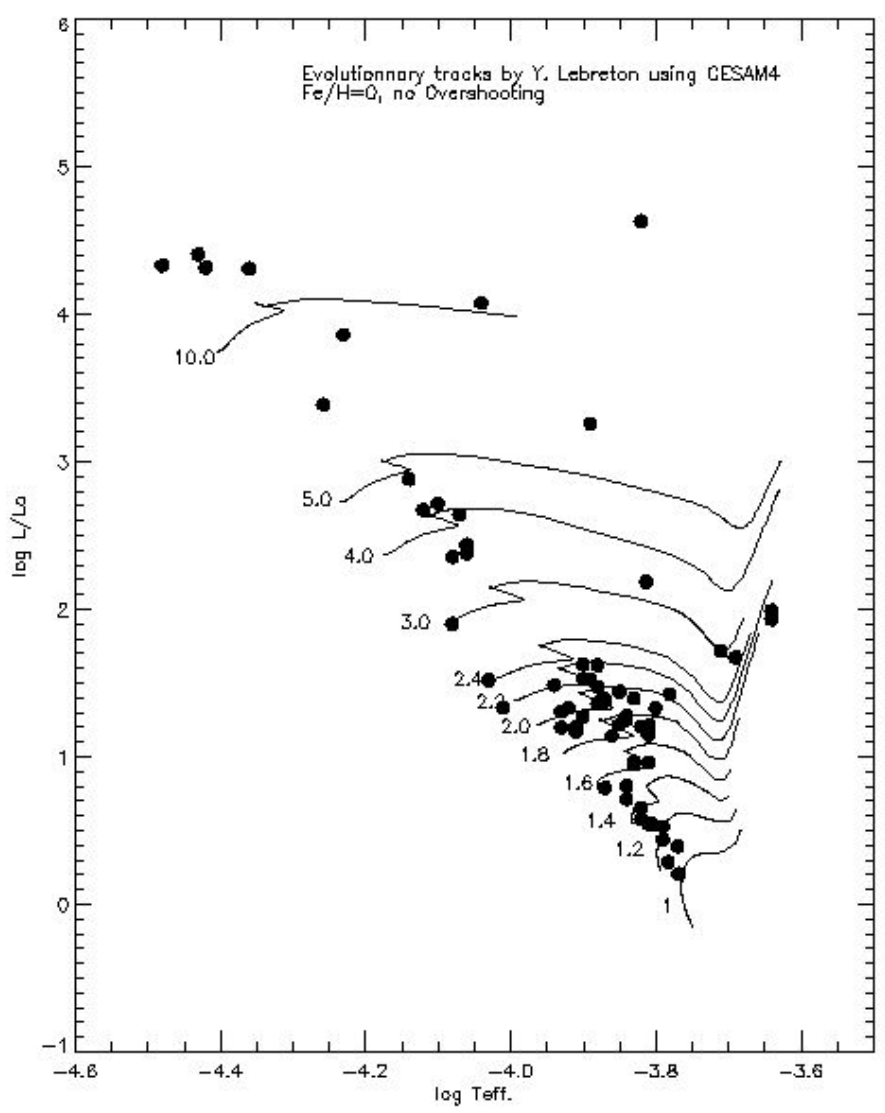

Figure 1: HR diagram of the targets to be observed during the Initial Run and Long Runs

\section{Detection level and precision on frequency measurement}

A major aspect of the CoRoT performance is the possibility to detect very low amplitude oscillations. In order to give a flavour of this, we use a statistical test proposed by Appourchaux et al. (2000): $r \simeq \ln (T)+\ln (\Delta)-\ln (P)$, where $T$ is the total duration of the observations (in seconds), $\Delta$ is the range of frequency searched for oscillation peaks (in $\mathrm{Hz}$ ) and $P$ is the probability to get at least one peak due to noise larger than $r$ times the local mean, in the power spectrum.

For solar-like oscillations, assumed to have lifetimes shorter than the observations, it was shown by Appourchaux (2004) that the test can be refined and optimized to better take into account the fact that the mode profile is resolved. But for simplicity, we here will use the same test as previously described, giving a bottom line for detection performance.

As by Michel et al. (2006a), for cases illustrative of a solar-like pulsators, the 1- $\sigma$ precision on frequency determination is estimated following Libbrecht (1992), while for the cases illustrative of classical pulsators, the estimate of the precision is given following Koen (1999). 


\section{Solar-like pulsators}

The case of solar-like pulsators has already been considered by Michel et al. (2006a) for CoRoT Long Runs. These authors selected two objects. On one hand, HD $49933\left(m_{V}=5.7\right)$, to be observed during LRA1, is representative of the best (brightest) candidates. On the other hand, HD $49385\left(m_{V}=7.9\right)$, also to be observed during LRA1, is representative of solar-like pulsators candidates for which, according to current theoretical amplitude estimates, one can expect detection of a significant number of peaks, but no guarantee of a high precision of the frequency measurement.

Simulations presented by Michel et al. (2006a) show that for HD 49933, the expected precision on frequency measurements goes from $\sim 0.15 \mu \mathrm{Hz}$ to $\sim 0.3 \mu \mathrm{Hz}$ when granulation noise estimate is taken into account. For HD 49385, the expected precision is about $0.4-$ $0.5 \mu \mathrm{Hz}$ with a lower relative influence of the potential contribution of granulation noise.

\section{During an Initial Run}

HD 49933 is also scheduled for Initial Run IR1, to be observed during 60 to 80 days at the very beginning of the observational program. The synthetic power spectrum corresponding to the simulation of a 60-day run on HD 49933 is presented in Fig. 2. For the photon noise only and for the photon noise plus the granulation noise contributions, we compute a $99 \%$ confidence level of detection following the statistical test described previously, with $T=60$ days, $\Delta=5 \mathrm{mHz}$, and $P=1 \%$. We notice that even with the granulation noise as estimated here, a large amount of the oscillation peaks is detected with a confidence level higher than $99 \%$.

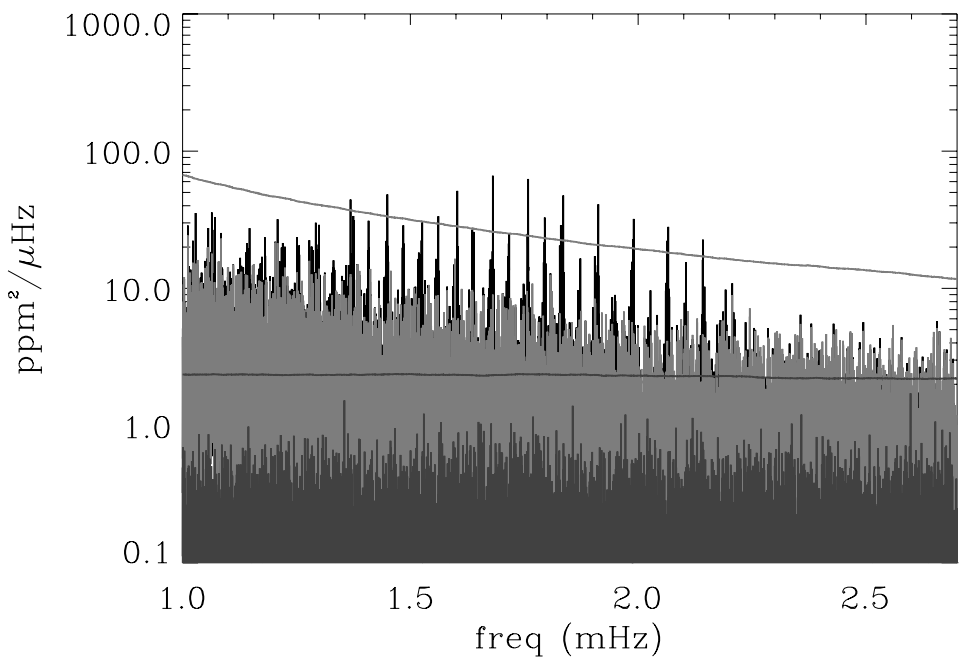

Figure 2: Simulation of the power spectrum expected for HD 49933 (observed during 60 days), including photon noise (dark grey), and granulation noise (light grey) estimates as described in the text. The $99 \%$ confidence levels of detection are drawn as lines associated with the photon noise only (dark grey), and the photon noise plus granulation noise (light grey). 
Figure 3 illustrates the expected precisions estimated following Libbrecht (1992) and for different cases:

a) The reference case: the precision of frequency determination is established considering only photon noise and taking $1 \mu \mathrm{Hz}$ as a fixed value for line widths. The excitation rates are computed following Samadi \& Goupil (2001).

b) As case a, but line widths are from Houdek et al. (1999).

c) As case b, but granulation noise contribution is also considered.

The results presented in Fig. 3 can be directly compared with those obtained for a 150-day run (Michel et al. 2006a, Fig. 5). As could be expected, the present precisions are significantly larger in all cases a, b and c. They however remain below $\sim 0.5-0.6 \mu \mathrm{Hz}$.
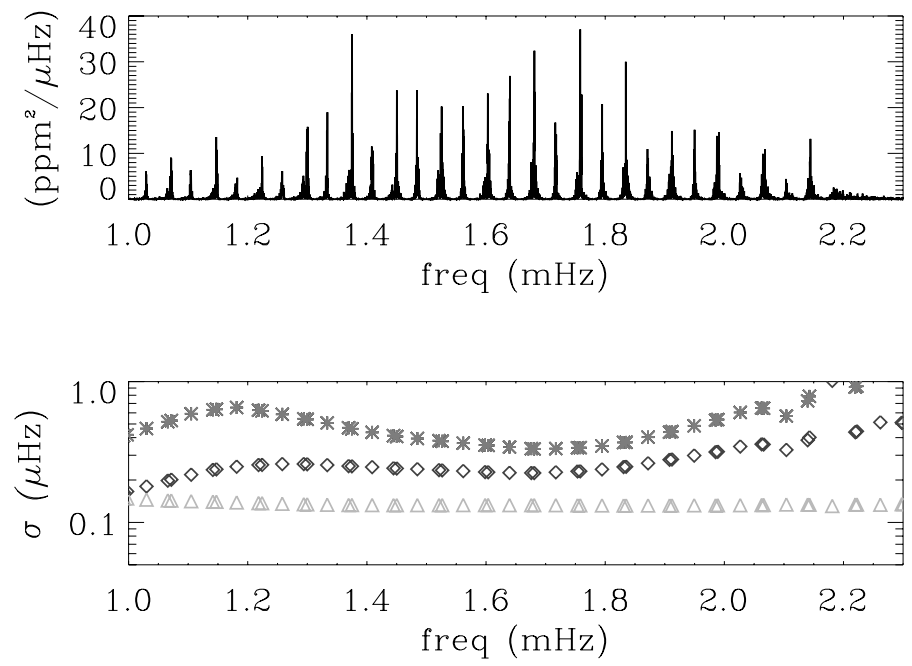

Figure 3: Lower panel: simulation of the pure seismic signal expected for HD 49933. Upper panel: Estimates of the 1- $\sigma$ precision on the determination of eigenfrequencies, for cases $a, b$ and $c$ (resp. triangles, diamonds, stars) as described in the text.

\section{Classical pulsators}

In order to illustrate the expected performance for classical pulsators, we have selected the example of HD $49294\left(m_{V}=7, v \sin i=111 \mathrm{~km} / \mathrm{s}\right)$, located in the $\delta$ Scuti instability strip, but for which preparatory surveys have not revealed variability at the mmag level (Poretti et al. 2005). Contrary to solar-like pulsations, classical pulsators show auto excited pulsations and since eventual amplitude and phase variations are still an open question it seems representative enough to use the work hypothesis that these oscillations are constant in amplitude and coherent in phase over a time span longer than the CoRoT observations. In addition to this, there is no theoretical prediction either of amplitudes for these objects. Observations from the ground reveal modes with amplitudes down to the detection threshold $10^{-3}$ (1000 ppm), occasionally a few hundreds of ppm.

For our simulation, we thus decided to consider arbitrary amplitudes of $100 \mathrm{ppm}$, i.e. peaks below what is currently detected from the ground. 


\section{During a Long Run}

Figure 4 illustrates such a simulation for HD 49294, a relatively bright object $\left(m_{V}=7\right)$. Here again, photon noise alone is represented in dark grey, while the photon noise plus granulation noise estimate is represented in light grey. In each case, the $99 \%$ confidence level for detection is represented by a line. It is computed following the previous definition, for $T=150 \mathrm{~d}, \Delta=1 \mathrm{mHz}$, and $P=1 \%$. Peaks above this limit are attributed to the star with a probability higher than $99 \%$.

The present case illustrates that here again, for bright objects, granulation noise, as estimated here, might be an important component for the final performance. Considering photon noise only, the $99 \%$ confidence level for detection is around $2 \mathrm{ppm}$, while including the granulation noise contribution, it is about $10 \mathrm{ppm}$ in the domain of interest. This still corresponds to a gain by a factor larger than 50 to 100 compared with what is currently obtained from the ground.

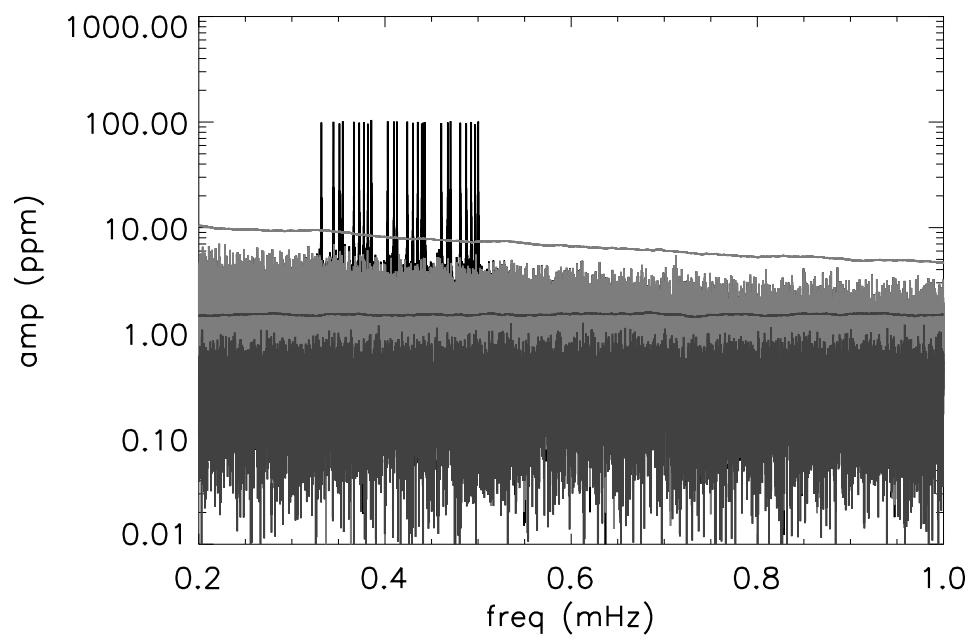

Figure 4: Simulation of the power spectrum expected for HD 49294, $m_{V}=7$, (observed during 150 days), including photon noise (dark grey), and granulation noise (light grey) estimates as described in the text. The $99 \%$ confidence level of detection are drawn as lines associated with photon noise only (dark grey), and photon noise plus granulation noise (light grey).

Besides the detection of the modes, it is interesting also to investigate the precision expected on frequency measurement for the detected modes. Following Koen (1999), all modes detected above the $99 \%$ confidence level are expected to have their frequency measured with a precision better than $\sim 1 /(10 T)$, where $T$ is the duration of the run (in seconds). In the present case, this means that all the peaks detected above the $99 \%$ confidence level will have their frequency measured with a precision better than $0.01 \mu \mathrm{Hz}$. This confirms the conclusion by Michel et al. (2006b) that such data will provide very valuable material for time/frequency analysis. 
For a fainter object during a Short Run

If HD 49294 were fainter (e.g. $m_{V}=9.5$, at the faint edge of the range of magnitudes considered in the Seismo field) and observed during a Short Run (20 days), the simulation provides the results shown in Fig. 5. We see that the detection threshold is between 10 and $20 \mathrm{ppm}$ if only photon noise is considered and around $30 \mathrm{ppm}$ including the present granulation noise estimate. Here again, this represents a significant gain (larger than 10) compared with current data. Following the same prescriptions as in the previous sections, but with $T=20 \mathrm{~d}$, the precision on frequency measurement is expected to be better than $0.065 \mu \mathrm{Hz}$ for the modes above the detection threshold. These results confirm the great interest of observing classical pulsators, even rather faint ones, during long and short runs.

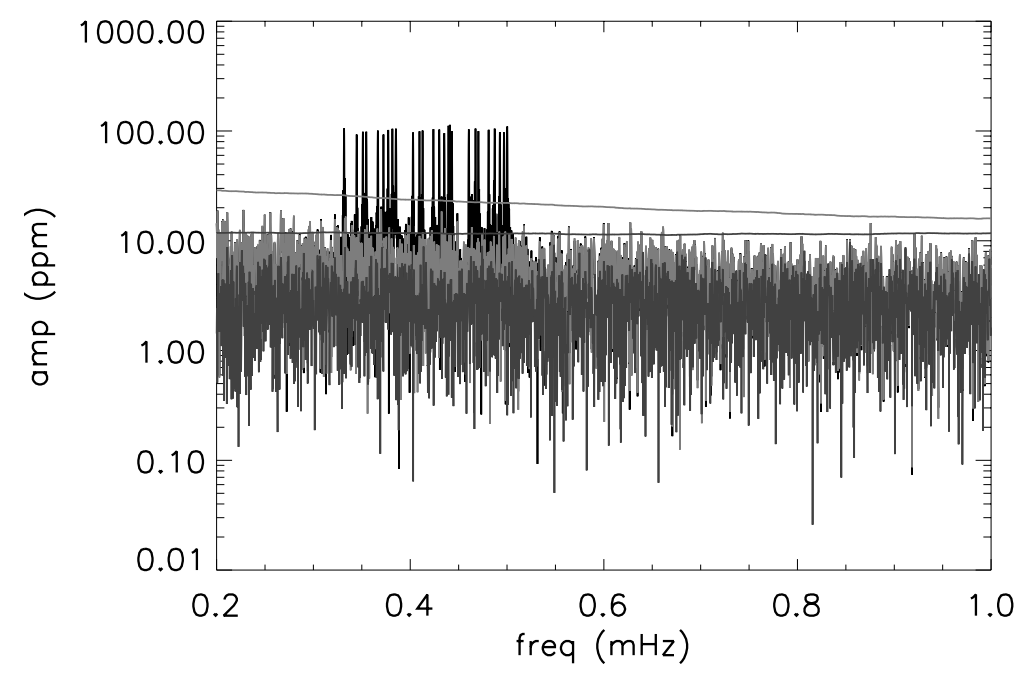

Figure 5: Same as Fig. 4, but for $m_{V}=9.5$ observed during a Short Run (20 d)

\section{Conclusion}

CoRoT is about to be launched and to bring a wealth of data expected to reveal a unique sight on stellar oscillations. It is our conviction that it will contribute to answer several open questions and lead to reconsider several aspects of the field of stellar structure and evolution, with consequences in numerous connected fields of research. Since the leading objective of this meeting was to discuss how to organize the future of stellar seismology, we have found it appropriate to illustrate, as precisely as possible at this stage, what will be the contribution of CoRoT data to this future, in terms of star sample and expected characteristics of the data.

\section{References}

Appourchaux T., Fröhlich C., Andersen B., et al., 2000, ApJ, 538, 401

Appourchaux T., 2004, A\&A 428, 1039

Baglin A., Michel E., Auvergne M., et al., 2006, in Fletcher K., ed., SOHO 18/GONG 2006/HelAs I: Beyond the spherical Sun. ESA SP-624, Noordwijk, p. 34.1 
Baudin F., Samadi R., Appourchaux T., Michel E., 2006, in Fridlund M., Baglin A., Conroy L., Lochard J., eds, 2006, The CoRoT Mission, Pre-Launch Status, Stellar Seismology and Planet Finding. ESA-SP 1306, Noordwijk, p. 403

Fridlund M., Baglin A., Conroy L., Lochard J., eds, 2006, The CoRoT Mission, Pre-Launch Status, Stellar Seismology and Planet Finding. ESA-SP 1306, Noordwijk

Harvey J., 1985, in Rolfe E., Battrick B., eds, Future Missions in Solar, Heliospheric and Space Plasma Physics. ESA-SP, 235, Noordwijk, p. 199

Houdek G., Balmforth N. J., Christensen-Dalsgaard J., Gough D. O., 1999, A\&A, 351, 582

Koen C., 1999, MNRAS, 309, 769

Libbrecht K. G., 1992, ApJ 387, 712

Michel E., Samadi R., Baudin F., et al., 2006a, Mem. Soc. Astron. Ital., 77, 539

Michel E., Baglin A., Auvergne M., et al., 2006b, in Fridlund M., Baglin A., Conroy L., Lochard J., eds, 2006, The CoRoT Mission, Pre-Launch Status, Stellar Seismology and Planet Finding. ESA-SP 1306, Noordwijk, p. 39

Michel E., Deleuil M., Baglin A., 2006c, in Fridlund M., Baglin A., Conroy L., Lochard J., eds, 2006, The CoRoT Mission, Pre-Launch Status, Stellar Seismology and Planet Finding. ESA-SP 1306, Noordwijk, p. 473

Poretti E., Alonso R., Amado P. J., et al., 2005, AJ, 129, 2461

Samadi R., Goupil M. J., 2001, A\&A, 370, 136 